\title{
Stability of Systems to Control Upright Postures in Stabilometry
}

\section{Hiroki Takada ${ }^{1,2 *}$}

${ }^{1}$ Graduate School of Engineering, University of Fukui, Fukui 910-8507, Japan

${ }^{2}$ Graduate School of Medicine, Aichi University of Medicine, Nagakute 480-1195, Japan

Keywords: Body sway; Stabilogram; Romberg's posture; Stochastic Differential equation (SDE); Temporally averaged potential function

\section{Background and Objective}

The author introduced the stabilometry to evaluate sports and doping effects that are effective as a countermeasure for a broad range of problems in our daily lives [1]. A countermeasure enables a recovery from some dysfunction or deconditioning. The clinical stabilometry is used to evaluate postural equilibrium utilized to keep the upright position in a specific time.

In upright postures, the body continuously moves in a unique and complex rhythm; the amplitude and frequency of this rhythm depend on various sensory and motor system functions [2]. The base supporting the body is an area surrounded by bilateral soles. To maintain a stable posture, it is necessary to control the spatial perpendicular line from the body's center of gravity within the narrow base [3-5]. The bilateral legs provide the ground reaction force required to support the body; however, the center of gravity continuously sways. This is because of the location of the head-upper limbs-trunk, which accounts for twothird of the body weight, is located at a distance that is two-third of the height from the floor. The human mechanism for maintaining upright postures is termed the righting reflex. Physiologically, the righting reflex is a body equilibrium function controlled by the involuntary regulatory system [6]. Elucidating the body's equilibrium function is essential for diagnosing symptoms related to impairment of the balance function, such as those of progressive cerebellar degeneration, basal ganglia disorder, and Parkinson's disease [7].

Stabilometry, which is performed as an equilibrium test, is useful for investigating the overall equilibrium function. Stabilometry is a simple test involving a 60 -se recording that starts when body sway stabilizes. Stabilometric methods and indices of body sway analysis, such as the total locus length and locus length per unit area, are used to increase the diagnostic value of stabilometry. The locus length per unit area represents the microchanges in postural control and is considered as a scale for functional evaluation of spinal proprioceptive postural control [8].

\section{Mathematical Models of Body Sway}

In stabilograms, variables $\mathrm{x}$ (cross direction) and $\mathrm{y}$ (longitudinal direction) are considered independent [9]. The linear stochastic differential equation (Brownian motion process) has been proposed as a mathematical model to describe body sway [10-12]. In order to describe the individual body sway, we especially show that it is necessary to extend the following nonlinear stochastic differential equations:

$$
\begin{aligned}
& \frac{\partial x}{\partial t}=-\frac{\partial}{\partial x} U_{x}(x)+w_{x}(t), \\
& \frac{\partial y}{\partial t}=-\frac{\partial}{\partial y} U_{y}(y)+w_{y}(t),
\end{aligned}
$$

Where $w_{x}(\mathrm{t})$ and $w_{y}(\mathrm{t})$ are pseudorandom numbers produced by white noise. The following formula describes the relationship $(\mathrm{z}=\mathrm{x}, \mathrm{y})$ between the distribution in each direction, $G_{z}(Z)$, and the temporal averaged potential constituting the stochastic differential equations, $U_{z}$ (Z) $[13]$.

$$
U_{z}(z)=-\frac{1}{2} \ln G_{z}(z)+\text { const }
$$

The variance of stabilograms generally depends on the temporal averaged potential function with several minimum values when it follows the Markov process without abnormal dispersion. Stochastic differential equations can represent movements within local stability with a high frequency component near the minimal potential surface, where a high density at the measurement point is expected.

\section{A Problem of the Stabilometry in Sports Medicine \& Doping Study \& Solution}

Stabilometry is generally performed in Romberg's or Mann's posture. Romberg's posture is an upright posture with the bilateral toes and heels together. Body sway increases in inversely to the area of the supporting base because these are unstable upright postures with a small support area; therefore, stabilometry in upright postures is not appropriate in subjects having difficulty maintaining a standing position for a certain amount of time, e.g. motion sickness, space flight deconditioning, and excessive exercise load [1]. Elderly persons and patients with Meniere disease may have difficulty sustaining this upright posture because their balance-retaining ability is impaired by aging and muscle weakness [14]. Romberg's posture with a narrow support area may increase the risk of falling, and hence, preventive countermeasures are necessary. Excluding unstable upright postures with a small support area or proposing surrogate postures for the stabilometry, we can prevent subjects from falling down on the floor.

Accordingly, changes in the standing position control system resulting from disturbance or abnormal body equilibrium function are reflected with greater sensitivity of the abovementioned unstable postures with a narrow support area. Stabilometry can usually detect the deterioration in the equilibrium function. Because the control system to maintain these postures is unstable, it is important to introduce the abovementioned surrogate postures to the stabilometry

- $\quad$ in which subjects are theoretically considered not to fall down

- which can detect the deterioration in the equilibrium function or

- In addition, few studies have succeeded in findings of potential functions (3) to control upright postures except for the Romberg's posture. Thus, we here in introduce

I. A posture with open toes and heels together

II. A posture with parallel feet to the stabilometry (Figures 1 and 2).

We herein compared histograms that were composed of all subject's

*Corresponding author: Hiroki Takada, Department of Human \& Artificial Intelligent Systems, Graduate School of Engineering, University of Fukui, Japan, 910-8507, Tel: 81-776-27-8795; E-mail: takada@u-fukui.ac.jp

Received December 13, 2013; Accepted December 16, 2013; Published December 23, 2013

Citation: Takada H (2013) Stability of Systems to Control Upright Postures in Stabilometry. J Sports Med Doping Stud 4: e141. doi:10.4172/2161-0673.1000e141

Copyright: ( 2013 Takada $\mathrm{H}$. This is an open-access article distributed under the terms of the Creative Commons Attribution License, which permits unrestricted use, distribution, and reproduction in any medium, provided the original author and source are credited. 
(a)

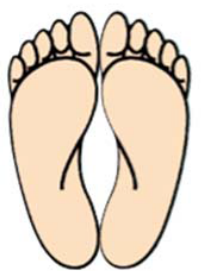

(b)

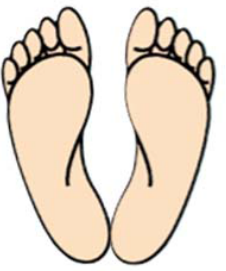

(c)

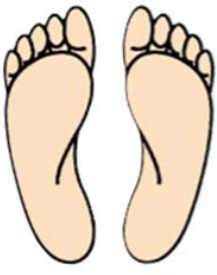

Figure 1: Arrangements of soles in each posture: (a) the Romberg's posture (b) a posture with open toes and heels together, and (c) a posture with parallel feet.

stabilograms. The frequency of an $\mathrm{x}$ coordinate near the origin was greater in the postures I and II than in the Romberg's posture. When the histograms for the Romberg's posture and the posture I were compared in the $\mathrm{x}$ and $\mathrm{y}$ directions, with open and closed eyes, using the chi-squared test, no significant differences were observed. However, there is a significant difference between histograms for the Romberg's posture and the posture II in the $\mathrm{x}$ direction. Thus, the system to control the upright posture I is regarded as the same system to maintain the Romberg's posture because potential functions to control upright postures can be obtained from the histograms in accordance with Equation (3) (Appendix) (Figure 2). However, the system to control the upright posture II is not regarded as the same system.

\section{Future Research Directions}

In previous studies on the body sway, stabilometry was generally performed in Figures 1 and 2 Romberg's posture. However, the balanceretaining ability decreases due to diseases (e.g., Meniere disease, sudden deafness accompanied by vertigo, and vestibular neuronitis), and the risk of falling is increased for patients in tests using Romberg's posture because it is an unstable upright posture. There is also an increased risk of falling when the technique is used for measurement of body sway, and course observation of vertigo and impaired balance function. If the risk of falling during the test can be reduced by performing stabilometry in the posture with heels together, additional studies are needed to further develop and validate the technique for application in medical practice.

Stabilograms indicated that the standing position in Romberg's posture was less stable in both young and elderly subjects [15]. Significant differences were also noted in several indices between Romberg's posture and the posture I in the younger participants although no significant differences were obtained in distributions for each component of the stabilograms. The former means that patterns and/or the indices for stabilograms can detect the postural change, sensitively. The later is consistent with the result of this paper; therefore the posture I may be used in stabilometry as a substitute for Romberg's posture in elderly persons.

The temporal averaged potentials in Romberg's posture were determined from the histograms using Equation (3) in the younger participants (Figure 2). The shapes of the potentials were more complex and tended to be nonlinear in the Romberg's posture and the lateral component $\mathrm{x}$ because coefficients of the regression polynomials (Appendix) were less than 0.9 in several cases (Tables 1 and 2). Multiple minimal points in the potential function have been obtained from each healthy young subject in accordance with the theory mentioned in the section 2 [13]. Especially in the posture II, two minimal points in the potential function might correspond to each center of the sole. In the next step, we will quantitatively examine whether distance between open toes changed the potential function in the posture I. (a)

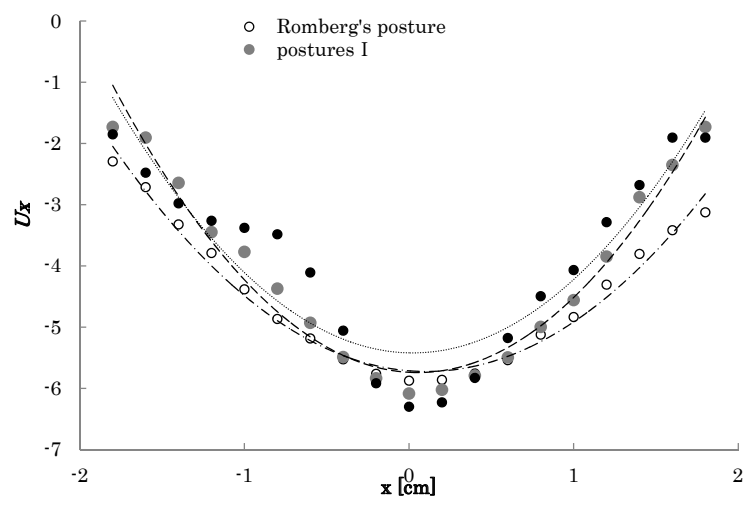

(b)
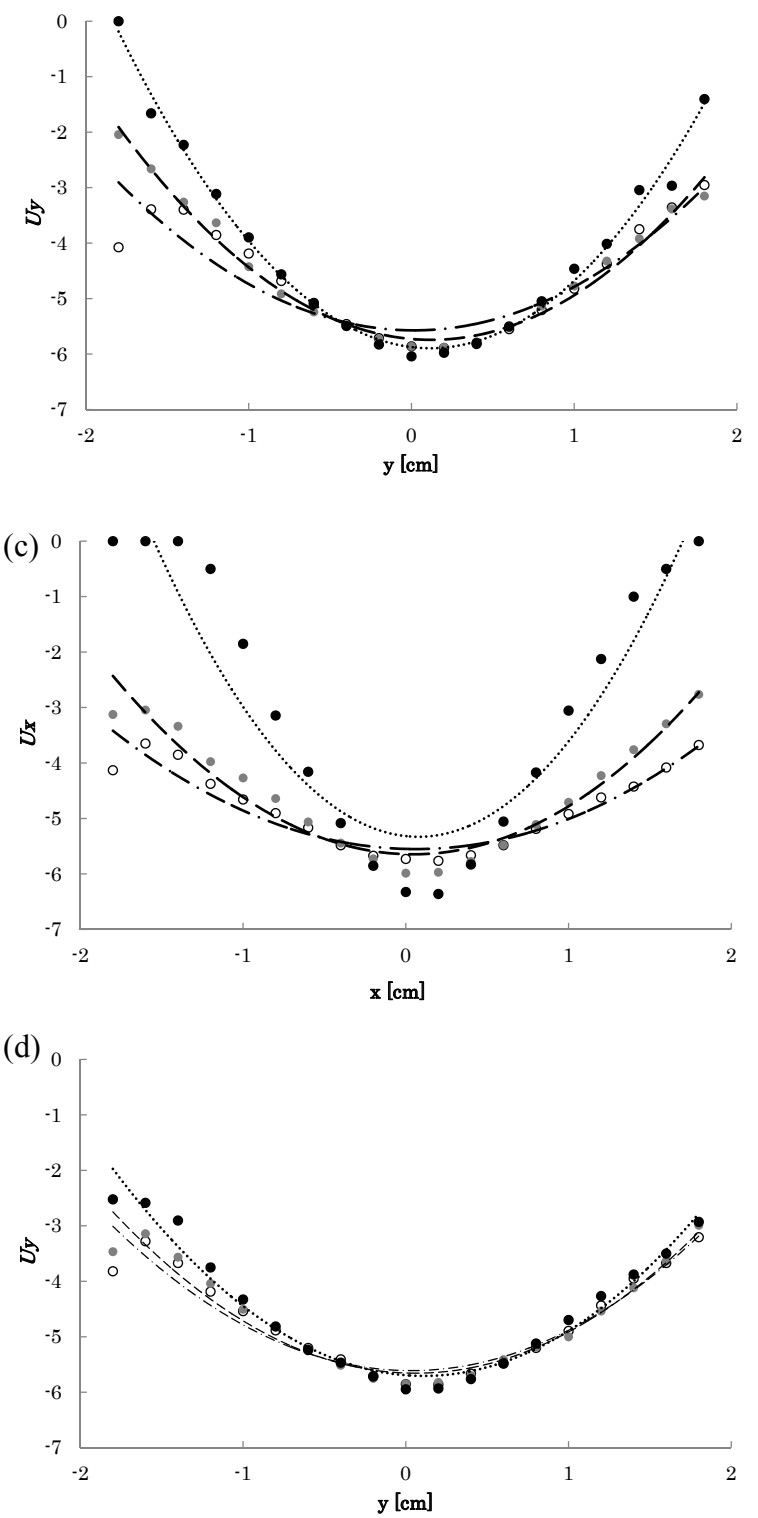

Figure 2: Temporal averaged potential function derived from stabilograms of all subjects in each posture with their eyes open (a), (c) and with their eyes closed (b), (d). 


\begin{tabular}{|c|c|c|c|c|}
\hline \multicolumn{5}{|c|}{ (a) component $x$} \\
\hline Romberg's posture & a & b & c & $R^{2}$ \\
\hline Posture I & 1.01 & -0.21 & -5.71 & 0.98 \\
\hline Posture II & 1.37 & -0.15 & -5.74 & 0.97 \\
\hline
\end{tabular}

(b) component $y$

\begin{tabular}{|l|l|l|l|l|}
\hline & a & b & c & $R^{2}$ \\
\hline Romberg's posture & 0.81 & -0.02 & -5.57 & 0.83 \\
\hline Posture I & 1.04 & -0.25 & -5.73 & 0.98 \\
\hline Posture II & 1.56 & -0.36 & -5.87 & 0.99 \\
\hline
\end{tabular}

Table 1: Coefficients of the potential function in each component: (a) latera direction $\mathrm{x}$, and (b) anterior/posterior direction, while subject's eyes were open.

(a) component $x$

\begin{tabular}{|l|l|l|l|l|}
\hline & a & b & c & $R^{2}$ \\
\hline Romberg's posture & 0.62 & -0.08 & -5.55 & 0.89 \\
\hline Posture I & 0.95 & -0.08 & -5.64 & 0.94 \\
\hline Posture II & 2.02 & -0.31 & -5.32 & 0.87 \\
\hline
\end{tabular}

\begin{tabular}{|c|c|c|c|c|}
\hline \multicolumn{5}{|c|}{ (b) component $y$} \\
\hline & a & $\mathrm{b}$ & c & $\mathrm{R}^{2}$ \\
\hline Romberg's posture & 0.78 & -0.05 & -5.61 & 0.91 \\
\hline Posture I & 0.84 & -0.10 & -5.65 & 0.94 \\
\hline Posture II & 1.02 & -0.22 & -5.69 & 0.96 \\
\hline
\end{tabular}

Table 2: Coefficients of the potential function in each component: (a) lateral direction $\mathrm{x}$, and (b) anterior/posterior direction, while subject's eyes were closed.

\section{Appendix Material}

The subjects used in the study included 17 young males (mean age \pm standard deviation: $22.5 \pm 0.87$ years) with no prior medical history of ear or nervous system disorders. The experiment was explained to the subjects and written consent was obtained before testing.

Stabilometry was performed in three postures using a random order: Romberg's posture, posture I and posture II. The stabilometer used was a gravicorder (GS3000, ANIMA Corp.), with a sampling frequency of $20 \mathrm{~Hz}$. The posture with heels together was an upright Romberg's posture with open tiptoes. After resting for $30 \mathrm{~s}$, consecutive 1-min body sway measurements were completed, with both open and closed eyes. In the tests with open eyes, subjects were instructed to look at a gazing point placed $2 \mathrm{~m}$ in front of them, at the eye level.

The center of pressure (COP) is the point on the area of the supporting base where the total sum of a pressure field acts. The projection of a subject's center of gravity in the $\mathrm{x}$ (right direction, designated as positive) and y (posterior direction, designated as negative) directions onto a detection stand is expected to be measured as a COP, an average of the pressure of both soles. The COP is traced for each sampling time, and the time series data is traced onto an $\mathrm{x}-\mathrm{y}$ plane. By connecting the temporally vicinal points, a stabilogram is created.

Histograms of the stabilograms obtained from tests on all postures were prepared. Each stabilogram was processed by subtracting the series mean from each time-series to set the center of the stabilogram at the origin $(0,0)$. A comparison of the histograms showed that the stabilogram distribution near the origin in the $\mathrm{x}$ direction increased in the postures I and II as compared to that in Romberg's posture, suggesting that stabilometry in the postures I and II reduces the body sway in the $\mathrm{x}$ direction.

Using Equation (3), potential functions to control upright postures can be obtained from the histograms of all subjects in this study (Figure 2). The potential function $U_{z}$ was here in regressed by a polynomial of degree 2:

$$
\hat{U}_{z}=a z^{2}+b z+c
$$

Whose coefficient of determination was sufficiently greater than 0.8 (Tables 1 and 2). Coefficients of the regression polynomials were also shown in Tables 1 and 2. The coefficients in the Equation (4) were estimated by the least square method. The lateral body sway in the posture II could be observed around the origin. The coefficient of degree 2, $a$ was smaller in the Romberg's posture than that in the posture II. Especially in the anterior/ posterior direction, this tendency was observed. Thus, the system to control upright posture II is regarded as a stable one, and variables $\mathrm{x}$ and $\mathrm{y}$ might not be ent in stabilograms observed during the posture II.

\section{References}

1. Takada H (2013) Stabilometry in Sports Medicine \& Doping Studies, Sports Medicine \& Doping Study 3: e129.

2. Okawa T, Tokita T, Shibata Y, Ogawa T, Miyata H (1995) Stabilometry: Significance of Locus Length per Unit Area (L/A), Equilibrium Research. 54: 296-306.

3. Gatev P, Thomas S, Kepple T, Hallett M (1999) Feedforward ankle strategy of balance during quiet stance in adults. J Physiol 514: 915-928.

4. Loram ID, Kelly SM, Lakie M (2001) Human balancing of an inverted pendulum: is sway size controlled by ankle impedance? J Physiol 532: 879-891.

5. Winter DA, Patla AE, Prince F, Ishac M, Gielo-Perczak K (1998) Stiffness control of balance in quiet standing. J Neurophysiol 80: 1211-1221.

6. Kaga K (1992) Memaino Kouzo (Structure of vertigo), Kanehara Shuppan, Tokyo, pp.23-26, pp.95-100.

7. Okawa T, Tokita T, Shibata Y, Ogawa T, Miyata H (1996) Stabilometry: Significance of Locus Length per Unit Area (L/A) in Patients with Equilibrium Disturbances, Equilibrium Research 55: 283-293.

8. Suzuki J, Matsunaga T, Tokumasu K, Taguchi K, Watanabe Y (1996) Handbook and Q\&A of Stabilogram, Equilibrium Research 55: 296-306.

9. Goldie PA, Bach TM, Evans OM (1989) Force platform measures for evaluating postural control: reliability and validity. Arch Phys Med Rehabil 70: 510-517.

10. Collins JJ, De Luca CJ (1993) Open-loop and closed-loop control of posture: A random-walk analysis of center of pressure trajectories. Exp Brain Res 95: pp.308-318.

11. van Emmerik RE, Sprague RL, Newell KM (1993) Quantification of postural sway patterns in tardive dyskinesia. Mov Disord 8: 305-314.

12. Newell KM, Slobounov SM, Slobounova ES, Molenaar PC (1997) Stochastic processes in postural center-of-pressure profiles. Exp Brain Res 113: 158-164.

13. Takada H, Kitaoka Y, Shimizu Y (2001) Mathematical index and model in stabilometry. Forma 16: 17-46.

14. Stevens JC, Patterson MQ (1995) Dimensions of spatial acuity in the touch sense: changes over the life span. Somatosens Mot Res 12: 29-47.

15. Yoshikawa K, Kinoshita F, Matsuura Y, Takada H (2013) Mathematical Models of Systems to Control Upright Postures in Stabilometry, IEEJ Transactions on Electronics, Information and Systems 133: 2200-2204. 